\title{
Chandler Wobble and Free Core Nutation of Neutron Stars
}

\author{
Alexander Gusev \& Irina Kitiashvili \\ Kazan State University, Kremlevskaya str.18, Kazan, 420008, Russia
}

\begin{abstract}
Extending the theory of core-mantle differential rotation of a planet, we have obtained the periods $P_{C W}$ and $P_{F C N}$ for different pulsars in the second approximation. We have investigated the dependence of these periods on the equation of state of neutron liquid, flatness of mantle and crust of the neutron star.
\end{abstract}

\section{Introduction}

The planetary system around pulsar B1257+12 (Wolszczan \& Frail 1992), located in the Virgo constellation at a distance of $300 \mathrm{pc}$, is most interesting, containing four known planets. Whether another three pulsars have planetary systems is still under discussion: PSR B0329+54 (1 planet), PSR B1620-26 (1 planet) and PSR B1828-11 (3 planets: A, B, C). The orbital periods $\left(P_{A}=0.68\right.$ yr, $\left.P_{B}=1.35 \mathrm{yr}, P_{C}=2.71 \mathrm{yr}\right)$ of the possible extrasolar planets around PSR B1828-11 follow the same 1:2:4 harmonic relationship of the Jovian satellites.

PSR B1828-11 shows long-term, periodic and correlated variations of the pulse shape and rate of slow-down with variations having periodicities of $\sim$ 1000,500 and 250 days (Stairs, Lyne \& Shemar 2000), probably resulting from precession of the spin axis caused by an asymmetry in the shape of the pulsar.

\section{Chandler Wobble and Free Core Nutation}

There are three areas potentially relevant for explaining anomalous times of arrival from a pulsar: those concerned with the neutron star interior, planetary bodies and free precession (Stairs et al. 2000). It is known that rotation of the terrestrial planets having rigid mantles and elliptical liquid cores is characterized by Free Core Nutation (Van Hoolst, Dehant \& Defraigne 1998; Gonzalez \& Getino 1997). Let us consider the free rotation of the pulsar, which has a rigid crust and an elliptical liquid mantle. The rotation of a two-layer pulsar with a liquid mantle must show two different polar modes (Petrova \& Gusev 2001): a wobble mode (like "Chandler Wobble", CW) and Free Core Nutation (FCN).

In the case of polar motion, from the corresponding Hamilton's equations we find the solution for the rotation of the mantle and the relative rotation of the core (Gusev \& Kitiashvili 2003). In this case the periods are

$$
P_{C W}=\frac{P_{P S R}}{2} \frac{A_{\text {crust }}}{A}\left(\frac{C}{A}-1\right)^{-1} ; P_{F C N}=-P_{P S R} \frac{A_{\text {crust }}}{A}\left(\frac{C_{\text {mantle }}}{A_{\text {mantle }}}-1\right)^{-1},
$$


where $A, C$ are moments of inertia of crust, mantle and whole pulsar.

Table 1. Modeling of flatness $\left(\epsilon \times 10^{-9}\right)$ from Chandler Wobble and Free Core Nutation of PSR B1828-11 ${ }^{a}$.

\begin{tabular}{lcccccccc}
\hline$P_{C W}, \mathrm{~d}$ & $\mathrm{R}$ & $\mathrm{BJ}$ & $\mathrm{MF}$ & TI1 & TI4 & TI5 & TI6 & TI7 \\
\hline 167 & 1.11 & 6.41 & 9.47 & 15.6 & 26.2 & 19.8 & 8.36 & 9.47 \\
250 & 0.74 & 4.28 & 6.33 & 10.4 & 17.5 & 13.2 & 5.58 & 6.33 \\
500 & 0.37 & 2.14 & 3.16 & 5.21 & 8.75 & 6.61 & 2.79 & 3.16 \\
1000 & 0.19 & 1.07 & 1.58 & 2.61 & 4.37 & 3.30 & 1.40 & 1.58 \\
\hline$P_{F C N}, \mathrm{~d}$ & $\mathrm{R}$ & BJ & MF & TI1 & TI4 & TI5 & TI6 & TI7 \\
\hline 167 & 2.23 & 12.8 & 18.9 & 31.2 & 52.4 & 39.6 & 16.7 & 18.9 \\
250 & 1.49 & 8.56 & 12.7 & 20.8 & 35.0 & 26.4 & 11.2 & 12.7 \\
500 & 0.74 & 4.28 & 6.33 & 10.4 & 17.5 & 13.2 & 5.58 & 6.33 \\
1000 & 0.37 & 2.14 & 3.16 & 5.21 & 8.75 & 6.61 & 2.79 & 3.16 \\
\hline
\end{tabular}

${ }^{a}$ The models of nuclear interaction are: Reid (R), Bethe-Johnson (BJ), approximation of an average field (MF), tensor interaction (TI).

We investigated the dependence of $P_{C W}$ and $P_{F C N}$ on the equation of state for a neutron liquid (Gusev \& Kitiashvili 2003), flatness of mantle and crust of the neutron star. Assuming that the observed variations of the period of PSR B1828-11 are caused by Chandler fluctuations and free core nutation of a liquid mantle we have obtained flatness of the mantle and crust of the pulsar (Table 1).

Detection of Chandler wobble and Free Core Nutation of the pulsar and its periods allow: (a) to decide on the physical nature of the pulsar mantle and core it is possible only for the crystal core; (b) to determine mantle and core radius and its flattening; (c) to determine density jump at the crust-mantle boundary and core-mantle boundary. For the theory of core-mantle differential rotation of the neutron star, we have obtained the period of CW and FCN of the liquid part of the neutron star. This was made in the frame of a Hamiltonian approach for description of the rotation of a two-layer deformable pulsar, having a rigid crust and a liquid mantle. Two modes of free pulsar libration are obtained in the case of polar motion from Hamilton's equations: the Chandler-like wobble with period $P_{C W}$, and a Free Core Nutation with period $P_{F C N}$.

\section{References}

Gonzalez, A-B., \& Getino, J. 1997, Celest. Mech. \& Dyn. Astr., 68, 139

Gusev, A., \& Kitiashvili, I. 2003, in Proc. Geon-Kazan-2003, ed. N. Petrova, Kazan Univ. Press, Vol. 3, 80

Petrova, N., \& Gusev, A. 2001, Cel. Mech \& Dyn. Astr., 80, 215

Stairs, I. H., Lyne, A. G., \& Shemar, S. L. 2000, Nature, 406, 484

Van Hoolst, T., Dehant, V., \& Defraigne, P. 1998, Phys. Earth \& Planetary Interiors, 117, 397

Wolszczan, A., \& Frail, D. A. 1992, Nature, 355, 145 